Отримано: 9 лютого 2019 р.

Прорецензовано: 25 лютого 2019 р.

Прийнято до друку: 1 березня 2019 р.

e-mail: oksanamartyniuk@ukr.net

DOI: $10.25264 / 2519-2558-2019-5(73)-42-45$
Oksana Martyniuk. Les moyens d'expression du refus en français moderne. Наукові записки Наиіонального університету «Острозька академія»: серія «Філологія». Острог: Вид-во НаУОА, 2019. Вип. 5(73), березень. С. 42-45.

Oksana Martyniuk,

УДК 811.133 .1 '373

docteur ès lettres du I cycle, maître de conférences à l'Université Nationale d'Europe Orientale Lessia Oukrä̈nka,

Chaire de Langues romanes et interlinguistique

\title{
LES MOYENS D'EXPRESSION DU REFUS EN FRANÇAIS MODERNE
}

L'article est consacré à l'étude des moyens d'expression verbaux et non verbaux du refus en français moderne. Les types de refus direct et indirect sont déterminés. Les particularités structurelles et sémantiques des déclarations avec la signification «refus» et leur charge pragmatique sont analysées.

Les outils linguistiques explicites et implicites pour exprimer le refus sont caractérisés. Les moyens d'expression du refus, non verbaux (gestes, expressions faciales et même le silence), qui jouent un rôle important dans le processus de communication sont considérés.

Dans l'aspect syntaxique, les déclarations avec une valeur du rejet peuvent être exprimées non seulement à l'aide des phrases narratives, mais aussi avec des phrases exclamatives, impératives et des questions rhétoriques. Il est prouvé que le refus doit être convaincant, correct et poli afin de ne pas provoquer une agression.

Mots-clés: refus direct et indirect, moyens d'expression verbaux et non verbaux du refus, refus explicite et implicite.

\author{
Мартинюк Оксана Миколаӥвна, \\ кандидат філологічних наук, дочент кафедри романських мов та інтерлінгвістики \\ Східноєвропейського національного університету імені Лесі Украйнки
}

\section{ЗАСОБИ ВИРАЖЕННЯ ВІДМОВИ В СУЧАСНІЙ ФРАНЦУЗЬКІЙ МОВІ}

Стаття присвячена дослідженню вербальних та невербальних засобів вираження відмови у сучасній франиузькій мові. Визначено типи прямої та непрямої відмови. Проаналізовано структурні та семантичні особливості висловлювань зі значенням “відмова" та їхне прагматичне навантаження. Охарактеризовано експліщитні та імпліцитні мовні засоби вираження відмови. Розглянуто невербальні засоби позначення відмови (жести, міміка, і навіть, мовчання), які відіграють значну роль у процесі комунікачії. У синтаксичному плані, висловлення зі значенням відмови можуть оформлятися не лише розповідними реченнями, але також окличними, наказовими та риторичними питаннями. Доведено, що відмова повинна бути переконливою, коректною та ввічливою, щоб не викликати агресії.

Ключові слова: пряма і непряма відмова, вербальні та невербальні засоби вираження відмови, експліцитна та імпліцитна відмова.

\author{
Oksana Martyniuk, \\ PhD. in Philology, Associate Professor of the Department of Romance Languages and Interlinguistics \\ Lesya Ukrainka Eastern European National University
}

\section{MEANS OF EXPRESSION OF REFUSAL IN THE MODERN FRENCH LANGUAGE}

The article is devoted to the study of verbal and non-verbal means of expressing refusal in modern French. Types of direct (using a particle no, a verb in a negative form; statements that indicate impossibility, unwillingness to do something, etc.) and indirect (may appear in the form of justification, deterrence, preference, principle, excuse / regret, avoidance) refusal are determined. Structural and semantic peculiarities of statements with meaning «refusal» and their pragmatic load are analyzed. Explicit and implicit language means of refusal have been characterized. Non-verbal means of refusal (gestures, facial expressions, and even silence), which play a significant role in the process of communication are considered. Knowledge of nonverbal language not only helps to better understand people, but also (and above all) to predict what reaction it caused before speaking, to feel the need for change to achieve the desired result.

In the syntactical aspect, statements with a value of refusal can be made not only with narrative sentences, but also with exclamative, imperative sentences and rhetorical questions.

It was that the strategy of refusing to execute something may be part of such tactics of communication:-declare incompetent; refer to the inability to respond to the request at this time - refuse without any motivation; to abstain from the answer, to promise nothing concrete; make it clear that you do not wish to complete the application.

It is proved that the refusal must be convincing, correct and polite, so as not to cause aggression.

Key words: direct and indirect refusal, verbal and nonverbal means of expression of refusal, explicit and implicit refusal.

À l>écrit comme à l>oral, il est souvent délicat de dire non. De fait, certaines maladresses peuvent blesser. Bien sûr, nous ne pouvons pas toujours satisfaire une demande, accepter telle ou telle offre et, donc, nous devons refuser.

Le refus exige une compétence socio-pragmatique très développée. Cet acte est en effet une déclaration d'hostilité, surtout lorsqu'il est prononcé à titre personnel: le refus d'une invitation, d'un cadeau, etc. est par métonymie le rejet de celui qui invite ou offre, ce qui est susceptible de susciter des agressions, voire de provoquer la rupture. Pour écarter tout risque le refus doit être correct, amical et convaincant.

Cette recherche s'inscrit à la fois dans le prolongement des études sur le refus en français (Trinh Duc T., Manno G., Berrier A., Mbow F., Mulo Farenkia B., Kerbrat-Orecchioni C., Pöll B. et d'autres) [1-9].

L'objectif de notre étude est d'analyser les caractéristiques structurelles et sémantiques des énoncés français contenant un "refus", et la signification pragmatique de telles déclarations dans le processus de communication. 
Lors de nos recherches, on a établi que le refus peut être exprimé à l'aide d'un énoncé direct ou indirect. On emploie souvent les stratégies directes suivantes: 1) «non»: - Je peux conduire? - Non; «jamais de la vie": -Vous devriez le rappeler. - Jamais de la vie! (Levy, 1, p. 417); 2) les verbes à la forme négative (ne pas): - Dorothy, jurez-moi sur l'honneur que ma grand-mère n'a pas fait modifier la toiture de cette maison! [...]. - Je ne jurerai pas!-répondit-elle furieuse (Levy, 4, 126); 3) «impossibilité»: Ta place est ici, avec moi! - Non, c'est impossible! (Musso, p. 465); 4) «pas d'intérêt/de volonté/ d'envie»: Je n'ai pas le temps ni l'envie d'en parler (Levy, 1, p. 519); 5) des formules explicites performatives: je refuse de déménager; je suis toutefois obligé de décliner; je me vois obligée de décliner.

Et pour adoucir le ton du refus direct, les mots suivants doivent être utilisés au début de la phrase: malheureusement, merci mais; désolé, très désolé, à mon grand regret, je le regrette sincèrement, désolé, je suis vraiment désolé, je m'excuse etc., par exemple: Merci d'avoir pensé à moi. J'aurais adoré être là mais je ne peux pas (6).

Parfois on trouve les expressions de refus dans les lettres d'affaires (refus de candidature, refus d'invitation). Mais il ne faut pas rédiger une lettre de refus sous l'emprise de la colère. Cela doit être un désaccord sans blesser: Cependant, malgré l'intérêt que suscite votre candidature, nous sommes au regret de de ne pas pouvoir répondre favorablement à votre demande (dynamique-mag. com); Nous avons bien reçu votre invitation. Malheureusement, nous avons le regret de vous annoncer que nous ne pourrons pas assister à... (8).

Dans le domaine de la communication d'affaires on peut identifier les tactiques langagières suivantes: - le refus catégorique: Nous sommes au regret de vous annoncer que votre candidature n'a pas été retenue pour le poste; - le refus empathique: J'entends bien votre point de vue, votre souhait..., cependant, je ne peux y répondre...;-le refus justifié: Après un examen attentif de votre lettre et de votre $C V$, nous avons le regret de vous informer que nous ne pouvons pas retenir votre candidature car votre profil ne répond pas aux critères exigés; - le refus sans réponse concrète: Merci, Monsieur, pour cet entretien, nous allons vous communiquer notre réponse; - le refus-compromis: Votre profil présente un réel intérêt, mais nous ne pouvons actuellement vous proposer un poste en relation avec vos compétences. Nous vous proposons, avec votre accord, de conserver votre CV dans notre base de données, afin, le cas échéant, de vous proposer un poste conforme à votre profil (9).

Le refus indirect peut apparaître sous forme de justification, dissuasion, préférence, principe, excuse / regret, évitement [6, p. 52].

Donc, le refus peut se justifier par les faits différents (par exemple: l'incertitude du remboursement de l'argent prêté, la gêne ou l'embarras d'accepter un prêt d'argent, l'évitement de la dette etc.): C'est très gentil de ta part mais je ne peux pas accepter, cela me gêne et ce n'est pas ton rôle.

La dissuasion est actualisée afin d'amener l'allocutaire à renoncer à son offre. Elle peut apparaitre solitairement ou en association avec d'autres types de refus et/ou d'actes subordonnés: Non ce n'est pas nécessaire. Je crois que je pourrai m'en sortir. La dissuasion s'exprime aussi au moyen des énoncés qui soulignent le caractère inopportun ( « je ne pense pas que ce soit une bonne idée ») ou précoce (« la vie continue j'aurai l'opportunité une autre fois ») de l'offre faite.

L'expression de la préférence s'énonce à l'aide des phrases du type "je préfère / je préfère ne pas X": Je préfère me débrouiller toute seule, je vais trouver une autre solution. Je préfère ne pas mélanger mes amis à ces affaires. Merci quand même (6).

La stratégie de l'allusion au principe est une forme de justification destinée à préciser que le refus n'est pas un signe de rejet de l'autre, mais qu'il obéit plutôt à certains principes de vie, à l'éducation reçue, etc.: Vraiment c'est trop gentil à toi, mais je me sens obligé de refuser, vois-tu ce n'est pas dans mes habitudes d'emprunter; ou l'autre exemple: -Désolée; chez nous, la tradition veut que Noël, c'est pour la famille seulement (7).

La demande d'excuse / l'expression du regret, cette stratégie consiste à souligner que l'on est conscient du désagrément qu'implique le refus pour la face du destinataire et qu'on refuse contre son gré: Ça ne m'intéresse pas, veuillez m'excuser, mais je me sens très bien dans mon poste actuel. Non merci quand même c'est dommage. Le sentiment de regret ici sert à indiquer que le locuteur sait que le refus est une information déplaisante.

La stratégie d'évitement, le locuteur y a recours pour éviter de se prononcer en faveur ou contre une offre: Je vais y réfléchir.

Parmi les unités lexicales avec une sémantique de refus, on a enregistré les verbes, tels que: décliner, refuser, haïr, rejeter, etc., par exemple: - L'anniversaire de mes dix ans, j'avais refusé son cadeau [....]. (Levy, 1, p. 137); Je dois donc respectueusement décliner l'invitation du comité (8). May refusa catégoriquement de remettre les pieds dans la tanière d'Édouard (Levy, 1, p. 348).

En outre, on emploie les adjectifs qualitatifs d'évaluation à connotation négative, qui révèlent la raison pourquoi un locuteur refuse une offre proposé ou un ordre (impossible, indifférent, terrible): - Tu peux me garder les enfants samedi? [...]. - Hélas, là ça sera impossible, mais un atre jour avec plaisir (Levy, 3, p. 127).

L'examen de la structure syntaxique des expressions avec le component "refus" a montré qu'en plus des phrases narratives, il en y a également des questions rhétoriques, des phrases exclamatives et des phrases impératives. Voici des exemples de refus sous la forme de questions rhétoriques: Ça t>embête que je fume chez toi? - Ça t’embête de fumer dehors? (7).

La raison du refus sous la forme de questions rhétoriques réside dans les sentiments négatifs et les expériences déplaisantes de l'orateur générés par l'information entendu ou par l'attitude envers l'auditeur.

L'expression de refus sous la forme des phrases exclamatives peut avoir la nuance négative: Comment! C'est idiot! Bof! Que c'est ennuyeux ! (7). Les phrases impératives ont aussi des particularités émotionnelles et pragmatiques: Est-ce que vous êtes l'auteur de ces lettres anonymes? Shylock a pointé du doigt la porte de son bureau. -Foutez-moi le camp, vous êtes ridicules! (Levy, 1, p, 548). Dans cet exemple la combinaison des gestes et de l'ordre a pour but le refus catégorique.

La stratégie consistant à refuser d'exécuter une demande peut s'inscrire dans de telles tactiques de communication: -se déclarer incompétent; faire référence à l'impossibilité de répondre à la demande en ce moment;- refuser sans aucune motivation; s'abstenir de la réponse, ne rien promettre de concret; indiquez clairement que vous ne souhaitez pas compléter la demande.

La personne peut refuser d'une manière explicite, donc, la réponse est claire: Non, cela ne sera malheureusement pas possible. Mais parfois le refus peut être représenté implicitement. Dans ce cas-là, le contexte et la situation de la communication jouent un rôle important. On peut affirmer que le contenu implicite (signification) est une information qui, sans expression directe, est déduite du contenu explicite (signification) des unités linguistiques résultant de leur interaction avec la connaissance du destinataire (le destinataire - le lecteur, l'auditeur) du texte, notamment avec des informations issues du contexte et de la situation de communication. 
Dans les repliques: - Vas-tu au cinéma? -Je dois me préparer pour les examens, la réponse a le contenu implicite (Non, je ne vais pas au cinéma) qui est compréhensible dans cette situation (le destinataire doit réaliser que la préparation sérieuse aux examens est incompatible avec la visite au cinéma).

Le moyen le plus efficace de communiquer des informations entre des personnes est le langage verbal. Cependant, la communication n'est pas toujours et pas seulement à l'aide de mots combinés dans la déclaration. Les gens utilisent un certain nombre de moyens non verbaux pour exprimer leurs opinions.

Les gestes, contrôlés ou incontrôlés, que nous faisons en parlant font partie du message: ils ponctuent la parole, la soulignent ou la renforcent. Ils peuvent aussi se substituer à la parole, ou lui donner un sens différent. Ces expressions non-verbales sont parfois déroutantes, elles peuvent avoir une signification différente selon le pays. En Grèce, un mouvement de la tête de droite à gauche signifie l'approbation, alors qu'en France il indique le refus.

La connaissance du langage non verbal permet non seulement de mieux comprendre des interlocuteurs, mais aussi (et surtout) de prédire quelle réaction il a provoquée avant de parler, de ressentir le besoin de changement pour obtenir le résultat souhaité.

Voici quelques exemples des expressions non-verbales qui désignent le refus:

- un hochement de tête de gauche à droite synonyme de refus ou de négation: Il a fait non de la tête ; et il est allé en chercher deux autres (Levy, 1, p. 409)

- on refuse poliment quelque chose ou une invitation en levant la main, la paume tournée vers l'extérieur, la tête légèrement inclinée (Non, merci !).

- la main semble jeter quelque chose par dessus l'épaule. On refuse ainsi de rendre service, ou une proposition qui nous semble exagérée (Des clous!).

- on exprime son incrédulité en tirant le bas de l'oeil à l'aide de l'index. Ce geste indique qu'on refuse de croire à quelque chose qui nous est dit (Mon oeil!).

Parfois le silence peut revêtir différents aspects de rejets: un refus, un rejet, un non-dialogue en prolongement de la peur, l'inacceptable, l'incompréhensible, l'horreur insurmontable. Le fait de ne pas répondre à quelqu'un, il n'y a rien de plus déstabilisant. Là on s'exprime par le silence, par exemple: - Est-ce que Josh a de l'humour? Je veux dire sous son faux sourire ravageur, est-ce qu'il a vraiment le sens de l'humour?

Luke considéra gravement Hope, et retourna à son microscope.

- J'aime bien parler à ton dos, reprit Hope, mais tu pourrais tout de même être un peu plus poli (Levy, 2, p. 28).

Dire non, c'est aussi une manière de s'exposer aux problèmes et aux critiques. Les causes viennent principalement d'un manque de communication. Beaucoup de non-dits, de préjugés qui s'installent, des stéréotypes, font qu'il faut remonter en amont pour mettre les choses au clair. Il faut savoir donner les raisons qui poussent à dire non. Il existe mille manières d'interpréter ce non. Il y a toujours un référent qu'il faut expliciter. Le non constitue, en quelque sorte, la fin d'un processus et le début d'un autre.

Les êtres sociaux que nous sommes choisissent souvent la réponse la plus socialement souhaitable. La conséquence est que nous nous mettons nous-mêmes en difficulté. Nous voulons éviter que notre interlocuteur se sente mal à l'aise. Pourquoi n'osons-nous pas formuler un refus? Les raisons de cette difficulté à refuser sont multiples et dépendent de notre éducation, notre vécu, notre environnement. Parfois nous avons peur de l'autorité, de devoir se justifier ou s'excuser tel un petit enfant; hantise de décevoir, de ne pas être aimé; crainte de faire de la peine ou de blesser; angoisse du conflit; besoin d'avoir la paix; manque de confiance en soi.

Mais il est essentiel de savoir dire non car accepter à contrecœur parce qu'on ne sait pas comment refuser sera inévitablement perçu par la personne en face. Cela lui laissera un goût âpre. Il y a également fort à parier pour que cela ait des retombées sur le stress et le travail de celui qui n'a pas su dire non. Finalement, ce dernier va nourrir une certaine rancœur vis-à-vis de son collègue. Amertume qu'il ne devra qu'à lui-même!

Ainsi, formuler adéquatement un refus c'est se respecter et respecter les autres. Cela évite toute manipulation, d'un côté comme de l'autre: les choses sont claires, chacun est à sa place. Formuler un refus est un moyen d'affirmer son existence et sa personnalité, ses valeurs, assumer ses décisions, etc. Dire non, c'est oser prendre pleinement sa place, mais aussi laisser à ses collaborateurs le droit d'oser, eux aussi, dire non et prendre leur propre place.

Pour conclure, il faut noter que quand vous refusez quelque chose c'est loin d'être de l'égocentrisme ou de l'égoïsme, mais bien une preuve d'authenticité et d'intelligence (dire «oui» alors que l'on pense et ressent profondément «non» serait une totale ineptie !). Il faut être juste envers vous-mêmes et vos collaborateurs, dire non avec conviction et sans agressivité permettra une communication fluide et positive.

La perspective de nos recherches ultérieures consiste à étudier les énonciations avec la signification du refus dans le langage des représentants d'autres cultures.

\section{Bibliographie}

1. Berrier, Astrid (2000), «Refus et politesse», in Wauthion, M. \& Simon, A.C. (dir.), Politesse et idéologie. Rencontres de pragmatique et de rhétorique conversationnelles, Louvain, Peeters, p. 145-155.

2. Brown, Penelope \& Levinson, Stephen (1987), Politeness: Some Universals in Language Usage, Cambridge, Cambridge University Press, $345 \mathrm{p}$.

3. Kerbrat-Orecchioni, Catherine (2005), Le discours en interaction, Paris, Armand Colin, 364 p.

4. Manno, Giuseppe (1999), «Savoir refuser à l'écrit: analyse d'un enchaînement non préféré de macro-actes», Journal of French Language Studies, 9, pp. 39-68.

5. Mbow, Fallou (2011), «L'acte de refus et le fonctionnement de la politesse dans les rencontres commerciales», LIENS, 14, p. $181-206$.

6. Mulo Farenkia, Bernard (2014), Speech acts and politeness in French as a pluricentric language. Illustrations from Cameroon and Canada, Münster, LIT Verlag, $224 \mathrm{p}$.

7. Mulo Farenkia, Bernard (2015), "Invitation refusals in Cameroon French and Hexagonal French", Multilingua - Journal of CrossCultural and Interlanguage Communication, 34(4), p. 577-603.

8. Pöll, Bernhard (2005), Le français langue pluricentrique? Étude sur la variation diatopique d'une langue standard, Frankfurt am Main, Peter Lang, $340 \mathrm{p}$.

9. Trinh Duc, Thai (1999), L'acte de refus: étude comparative interculturelle des commerces français et vietnamiens. Mémoire de DEA en sciences du Langage. Université Lumière Lyon 2. 


\section{Liste des ouvrages cités}

1. Levy M. La dernière des Stanfield. Paris: Robert Laffont, 2017. 464 p.

2. Levy M. L'Horizon à l'envers. Paris: Robert Laffont-Versilio, 2016. 418 p.

3. Levy M. Mes amis mes amours. Paris: Robert Laffont, 2006. 415 p.

4. Levy M. La prochaine fois. Paris: Robert Laffont, 2004. 280 p.

5. Musso G. La Fille de papier. Paris: XO éditions, 2010. 378 p.

6. Comment décliner. URL: https://www.manager-go.com/efficacite-professionnelle/savoir-dire-non.

7. 45 façons de dire non. URL: http: // www. coupdepouce.com.

8. Refus d'invitation. Modèles de lettres. URL: http: // pratique. Le parisien.fr.

9. Refus de candidature. URL: http: // www.dynamique-mag.com. 